J3eA, Journal sur l'enseignement des sciences et technologies de l'information et des systèmes, Volume 4, Hors-Série 1, 13 (2005)

DOI : http://dx.doi.org/10.1051/bib-j3ea:2005613

(C) EDP Sciences, 2005

Architectures segmentées d'alimentation à convertisseurs modulaires pour les actionneurs d'avion

Fonctionnement en modes dégradés

F. Meibody-Tabar ${ }^{1}$, B. Davat ${ }^{1}$, R. Meuret ${ }^{2}$ et S. Vieillard ${ }^{2}$

${ }^{1}$ GREEN-INPL, CNRS UMR 7037

${ }^{2}$ HISPANO-SUIZA, Groupe SNECMA 


\title{
Architectures segmentées d'alimentation à convertisseurs modulaires pour les actionneurs d'avion ; fonctionnement en modes dégradés
}

\author{
F. Meibody-Tabar*, B. Davat*, R. Meuret**, S. Vieillard** \\ * GREEN-INPL, CNRS UMR 7037 \\ ** HISPANO-SUIZA, Groupe SNECMA
}

\section{Résumé :}

Pour que les actionneurs électriques puissent remplacer leurs homologues hydrauliques ou mécaniques dans les avions, ils doivent non seulement développer les performances requises dans différentes conditions de fonctionnement, mais aussi être fiables. Pour atteindre ces objectifs il faut considérer tous les problèmes qui peuvent survenir dans la chaîne de conversion électromécanique d'énergie: du réseau électrique embarqué jusqu'aux différents composants d'actionneurs. Avec un actionneur mono-convertisseur mono-machine, un défaut sévère provenant du réseau, du convertisseur électrique ou de la machine peut à lui seul interrompre la conversion électromécanique. Une solution pour améliorer la fiabilité du système consiste à segmenter la puissance qui transite entre la ou les sources électriques et le récepteur mécanique. Cette modification structurelle apporte des degrés de liberté supplémentaires permettant le fonctionnement de l'actionneur en modes dégradés. Dans cet article, nous présentons deux architectures segmentées d'alimentation d'actionneurs motorisés par des machines à aimants, triphasée et double-étoile; nous montrons que sous réserve de la prise en compte de certaines contraintes dans la conception de ces machines, le fonctionnement en présence de différents types de défauts est envisageable.

\section{Introduction}

L'utilisation accrue d'actionneurs électriques dans les avions a indéniablement contribué d'une part à la réduction des coûts d'exploitation par des économies d'énergie, et d'autre part à l'accroissement de la sûreté du fonctionnement par la maîtrise instantanée des différentes variables sensibles du système. Pour améliorer davantage la sûreté du fonctionnement, un choix adapté de l'architecture des chaînes de conversion d'énergie électrique et de leurs composants s'avère indispensable. Une solution consiste à segmenter la puissance qui transite entre la source électrique et le récepteur en un ou plusieurs endroits. Cela permet de remplacer un convertisseur (électrique, électromécanique ou mécanique) par deux ou plusieurs convertisseurs de même nature. Cette modification structurelle apporte des degrés de liberté supplémentaires pouvant être utilisés pour obtenir soit une meilleure qualité de couple et/ou de courant absorbé côté réseau, soit une meilleure fiabilité liée à la possibilité de fonctionnement en modes dégradés $[1,2,5]$. Cependant, la segmentation de puissance des chaînes de conversion d'énergie conduit également à l'apparition de couplages de nature électrique, magnétique ou mécanique qui peuvent imposer certaines contraintes $[3,4]$. Celles-ci sont parfois sévères et peuvent en pratique interdire certaines associations convertisseursmachines ou imposer l'application de stratégies spécifiques pour le contrôle des grandeurs du système. Les travaux menés par les chercheurs de plusieurs laboratoires, participant au projet "Systèmes Multi-machine Multiconvertisseur" du GDR SDSE, ont permis de mettre au point un formalisme pour représenter les systèmes électromécaniques complexes et analyser les interactions entre ses différents éléments physiques $\mathrm{Ce}$ formalisme permet notamment de mettre en évidence les couplages dans une chaîne de conversion électromécanique d'énergie à architecture segmentée [3]. La commande de ces systèmes avec la prise en compte de différents couplages, fait actuellement l'objet de travaux communs au sein du GDR $\mathrm{ME}^{2} \mathrm{MS}[4,6]$.

Dans cet article, nous présentons deux architectures segmentées d'alimentation d'actionneurs munis de machines synchrones à aimants permanents double-étoile ou à trois phases. Pour ces machines, nous mettons en évidence les contraintes liées aux couplages magnétiques entre les étoiles ou entre les phases, et nous en déduisons les conditions nécessaires à la sûreté d'alimentation de ces machines par des onduleurs de tension.

Dans le but d'accroître le degré de fiabilité des actionneurs électriques, la segmentation de puissance, appliquée au départ au niveau de l'ensemble onduleur-machine, est ensuite étendue à la source (réseau-redresseur-filtre d'entrée). Certaines architectures d'alimentation respectant ce principe et étant issues des travaux menés au GREEN en collaboration avec la société HISPANO-SUIZA, sont présentées en dernière partie de l'article.

\section{I- Analyse de fonctionnement d'actionneurs mono- convertisseur mono-machine}

L'architecture classique d'alimentation d'un actionneur d'avion, composé d'un redresseur - d'un filtre LC- d'un onduleur et d'une machine, est présentée sur la figure 1. L'actionneur est généralement motorisé par une machine synchrone à aimants ; pour le contrôle de son couple deux capteurs de courant et un capteur de position sont nécessaires. Le point faible de cette architecture est qu'un défaut sur chacun des composants de la chaîne de conversion d'énergie entraîne la perte de contrôle (totale ou partielle) de l'actionneur. En pratique, le défaut peut concerner le réseau (microcoupures, court-circuit, ...), le redresseur (casse de diode), l'onduleur (casse de transistor ou de diode), la machine (court-circuit dans le bobinage ou démagnétisation des aimants) ou l'un des capteurs. Parmi ces défauts, ceux concernant l'onduleur et sa commande rapprochée ainsi que ceux du réseau peuvent être considérés comme étant les plus probables. Etant donné qu'il existe dans certains avions deux réseaux identiques, en cas de défaut sévère sur un réseau il est possible d'alimenter l'actionneur par le deuxième (Fig. 1), en prenant évidemment les précautions nécessaires. Par contre, en cas 
de défauts internes, le contrôle de l'actionneur est totalement ou partiellement mis en cause.

Pour atteindre la fiabilité requise, une solution consiste à prévoir deux actionneurs identiques entraînant la même charge mécanique; en cas de défaut interne d'un actionneur, le deuxième peut être activé. Cependant, cette solution impose le dédoublement de tous les composants de l'actionneur, bien que la probabilité d'apparition de défaut dans un élément soit fortement différente de celles des autres éléments. Une autre solution consiste à modifier l'architecture de la chaîne de conversion d'énergie en segmentant la puissance au niveau des organes à fort risque d'apparition de défaut.

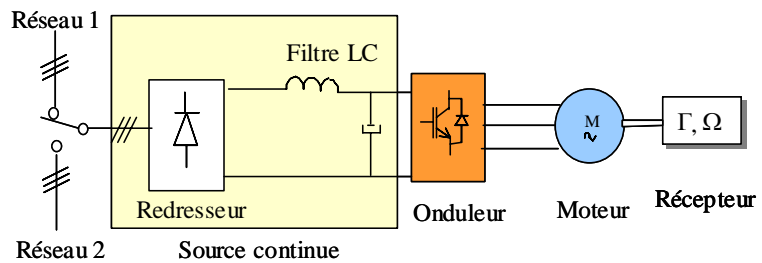

Figure 1 : Schéma d'un actionneur mono-convertisseur mono-machine

\section{II- Différentes approches de segmentation de puissance}

En partant d'une chaîne mono-convertisseur monomachine, deux approches de segmentation de puissance peuvent être envisagées :

- La première concerne uniquement le convertisseur électrique; on peut soit utiliser un convertisseur dont chaque composant est constitué de deux ou plusieurs composants en parallèle ou en série, soit remplacer le convertisseur par plusieurs convertisseurs modulaires en parallèle. La segmentation de puissance par la mise en parallèle (ou en série) des composants du convertisseur est inutile, car un défaut de court-circuit (ou circuit ouvert) dans un composant désactive également le contrôle des composants qui sont en parallèle (ou en série) avec le composant défectueux. La segmentation de puissance par la mise en parallèle de deux convertisseurs modulaires est possible mais pose d'autres problèmes, notamment dans le cas des onduleurs de tension. En effet, pour remplacer un onduleur de tension par deux onduleurs modulaires, afin d'éviter un courant de circulation élevé (court-circuit de la source continue), il est nécessaire d'utiliser des éléments inductifs (Fig.2). Comme ces éléments sont lourds et entraînent une chute de tension limitant les performances de la machine à vitesses élevées, leur présence diminue l'intérêt de cette solution pour les actionneurs d'avion.

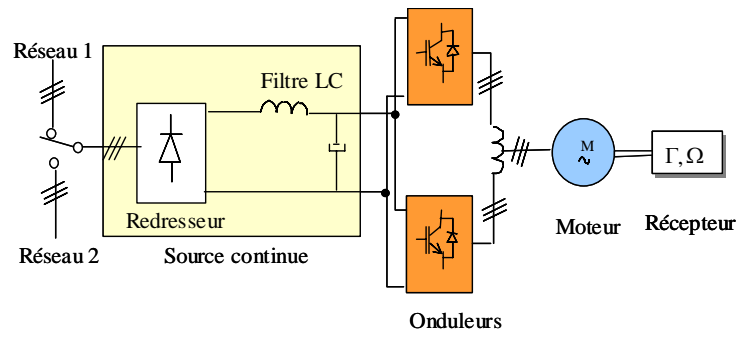

Figure 2 : Segmentation de puissance au niveau du convertisseur électrique.
- L'autre approche est basée sur la segmentation de puissance au niveau du convertisseur électrique tout en modifiant la structure du convertisseur électromécanique de sorte que les éléments inductifs externes (Fig. 2) puissent être évités sans qu'il y ait le risque d'un courant de circulation élevé [7]. Les deux structures suivantes, déjà étudiées au GREEN [1, 2], peuvent sous certaines conditions satisfaire cette exigence :

- La première structure utilise une machine doubleétoile dont chaque étoile est alimentée par un onduleur triphasé (Fig. 3) : à tension donnée de la source continue, la tension maximale imposée à chaque phase d'une machine double-étoile (Fig. 3) est identique à celle imposée à chaque phase d'une machine simple étoile (Fig. 1); pour la même puissance de sortie, le courant de phase d'une machine double-étoile est égal à la moitié du courant de phase d'une machine triphasée. En cas de défaut d'un onduleur, le bobinage étoile correspondant doit être déconnecté ; la machine peut ainsi fonctionner en mode dégradé avec une étoile alimentée par l'onduleur non-défectueux.

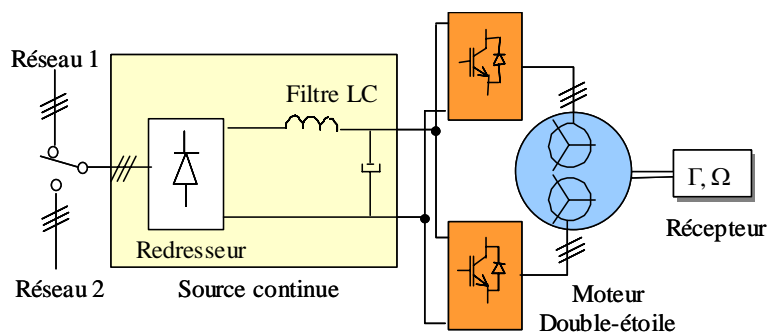

Fig. 3 : Machine double-étoile alimentée par deux onduleurs de tension

- La deuxième structure comporte une machine à aimants triphasée, alimentée par trois onduleurs monophasés (Fig. 4) ; les six bras des trois onduleurs monophasés peuvent être aussi regroupés en deux onduleurs classiques à trois bras (Fig. 5). Avec cette structure, l'amplitude du fondamental de la tension imposée à chaque phase peut atteindre le double de celle qu'impose un onduleur triphasé à une phase d'une machine triphasée montée en étoile. Ainsi, à puissance donnée, on peut diviser par deux le courant de phase et donc le courant dans des composants des onduleurs : afin de tirer les mêmes performances externes (couple, vitesse, pertes), le nombre de spires des bobines statoriques doit être doublé en divisant par deux la section de leurs conducteurs. En cas de défaut d'un onduleur monophasé, plusieurs stratégies de fonctionnement en mode dégradé peuvent être envisagées [2].

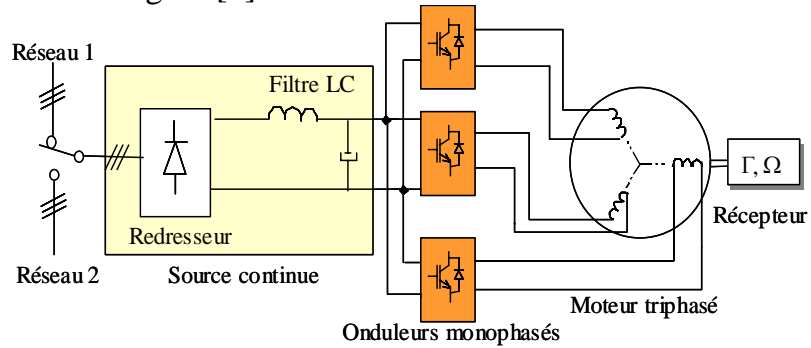

Figure 4 : Machine triphasée alimentée par trois onduleurs monophasés. 


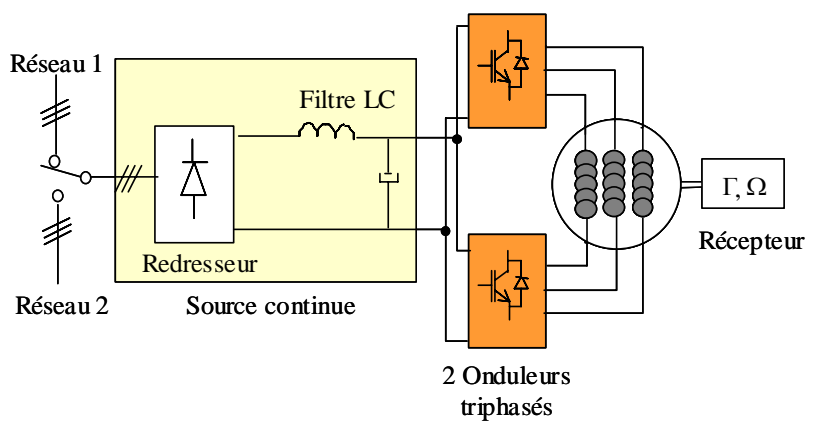

Figure 5 : Machine triphasée alimentée par deux onduleurs triphasés.

Les deux structures segmentées, comportant soit une machine double-étoile (Fig.3) soit une machine triphasée (Fig. 4 ou Fig.5), autorisent un fonctionnement en présence de défaut d'un onduleur. On bénéficie ainsi des avantages d'une segmentation de l'alimentation, d'une redondance et de l'utilisation de convertisseurs modulaires de puissance réduite, tout en ayant des machines dont la conception est relativement classique. Cependant, ces avantages sont acquis au prix de l'augmentation de l'ordre du système. En effet, une machine à trois phases montées en étoile n'a que deux courants indépendants, tandis qu'il y a respectivement quatre courants indépendants dans une machine doubleétoile et trois dans une machine à trois phases indépendamment alimentées. Dans ce qui suit nous présentons les modèles de ces machines et montrons que le contrôle efficace de leurs courants et donc la sûreté de leurs alimentations sont soumises à des conditions qu'il est nécessaire de satisfaire.

\section{III- Modélisation et sûreté de fonctionnement de machines à structure segmentée d'alimentation}

Compte tenu de l'application, nous ne considérons ici que les machines synchrones à aimants permanents (MSAP) à structure segmentée d'alimentation: machines synchrones double-étoile (MSDE) et machines à trois phases indépendamment alimentées. Pour ces machines, nous faisons également les hypothèses supplémentaires suivantes :

- le circuit magnétique n'est pas saturé,

- le phénomène d'amortissement au rotor est négligeable

- les pertes par hystérésis et par courants de Foucault au stator sont négligées ou reportées à l'extérieur du modèle,

- les courants capacitifs sont négligeables,

- la répartition des conducteurs des bobines statoriques et donc les fém de phases sont sinusoïdales,

- le rotor est supposé lisse du point de vue magnétique,

\section{III.1 MSAP double-étoile}

La machine étudiée est une machine synchrone formée d'un stator constitué de deux enroulements triphasés montés en étoile et décalés entre eux d'un angle électrique $\gamma$ (Fig. 6). L'excitation de la machine est assurée par des aimants permanents montés sur la surface du rotor. Pour établir son modèle, on appelle :

- $G_{k}$ une grandeur associée à la $k^{\text {ème }}$ phase de la première étoile (fém $\mathrm{e}_{\mathrm{k}}$, courant $\mathrm{i}_{\mathrm{k}}$ ou tension $\mathrm{v}_{\mathrm{k}}$ ),
- $\mathrm{G}_{\mathrm{k}}^{\prime}$ une grandeur associée à la $\mathrm{k}^{\text {ème }}$ phase de la deuxième étoile (fém $\mathrm{e}_{\mathrm{k}}^{\prime}$, courant $i_{\mathrm{k}}^{\prime}$ ou tension $\mathrm{v}_{\mathrm{k}}^{\prime}$ ),

- $p$ le nombre de paire de pôles,

- $r$ la résistance d'un enroulement,

- $l_{p}$ l'inductance propre d'un enroulement,

- $m \cdot \cos (\xi)$ la mutuelle entre deux enroulements du stator, décalés entre eux d'un angle électrique $\xi$.

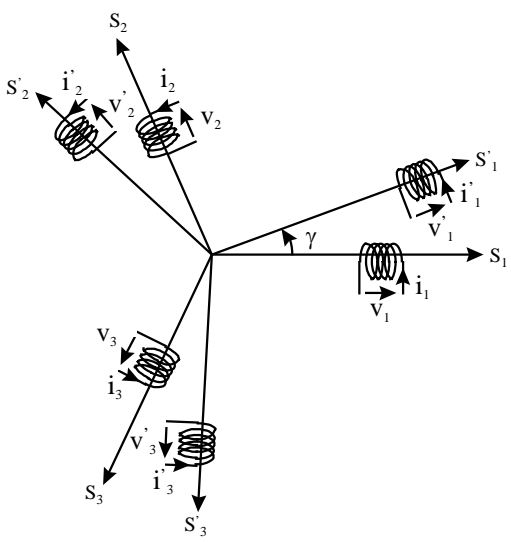

Figure 6 : Représentation des enroulements des différentes phases des deux étoiles statoriques.

L'équation de la tension de la $\mathrm{k}^{\mathrm{ème}}$ phase de chaque étoile s'écrit :

$\left\{\begin{array}{l}v_{k}=r \cdot i_{k}+\frac{d \phi_{k}}{d t}+e_{k} \\ v_{k}^{\prime}=r \cdot i_{k}^{\prime}+\frac{d \phi_{k}^{\prime}}{d t}+e_{k}^{\prime}\end{array} \quad\right.$ avec : $\mathrm{k}=1,2,3$.

où $\phi_{\mathrm{k}}$ et $\phi_{\mathrm{k}}^{\prime}$ ont pour expressions :

$\left\{\begin{array}{l}\phi_{k}=\left(l_{p}+\frac{m}{2}\right) \cdot i_{k}+\frac{3 \cdot m}{2} \cdot \cos (\gamma) \cdot i_{k}^{\prime}-\frac{m \cdot \sqrt{3}}{2} \cdot \sin (\gamma) \cdot\left[i_{k+1}^{\prime}-i_{k+2}^{\prime}\right] \\ \phi_{k}^{\prime}=\left(l_{p}+\frac{m}{2}\right) \cdot i_{k}^{\prime}+\frac{3 \cdot m}{2} \cdot \cos (\gamma) \cdot i_{k}+\frac{m \cdot \sqrt{3}}{2} \cdot \sin (\gamma) \cdot\left[i_{k+1}-i_{k+2}\right]\end{array}\right.$

Les forces électromotrices $\mathrm{e}_{\mathrm{k}}$ et $\mathrm{e}_{\mathrm{k}}^{\prime}$ sont définies par :

$\left\{\begin{array}{l}e_{k}=-\psi_{f} \cdot \omega \cdot \sin \left(\theta-(k-1) \cdot \frac{2 \pi}{3}\right) \\ e_{k}^{\prime}=-\psi_{f} \cdot \omega \cdot \sin \left(\theta-(k-1) \cdot \frac{2 \pi}{3}-\gamma\right)\end{array}\right.$

avec : $\quad \omega=\dot{\theta}=p \Omega$.

$\theta$ est l'angle électrique désignant la position du rotor par rapport à l'axe de la première phase de la première étoile ; $\psi_{f}$ est la valeur maximale du flux total des aimants à travers chaque bobine statorique.

Comme les courants dans les phases statoriques n'ont pas de composante homopolaire (la somme des courants de chaque étoile est nulle), chaque étoile statorique peut être remplacée par un bobinage diphasé équivalent. Afin d'exprimer, dans le même référentiel, les différentes grandeurs associées aux deux étoiles, on applique à cellesci la transformation "triphasée-diphasée" suivante :

$\left\{\begin{array}{l}{\left[G_{\alpha, \beta}\right]=T_{32}^{t} \cdot\left[G_{1,2,3}\right]} \\ {\left[G_{\alpha, \beta}\right]=P(-\gamma) \cdot T_{32}^{t}\left[G_{1,2,3}^{\prime}\right]=\left[T_{32}^{t}(\gamma)\right]\left[G_{1,2,3}^{\prime}\right]}\end{array}\right.$ 
où $\mathrm{T}_{32}^{\mathrm{t}}(0)$ est la transformation de Concordia et $\mathrm{T}_{32}^{\mathrm{t}}(\gamma)$ est la transformation de Concordia suivie d'une rotation d'un angle $\gamma$. Nous avons donc :

$$
\left[T_{32}^{t}(\gamma)\right]=\sqrt{\frac{2}{3}} \cdot\left[\begin{array}{ccc}
\cos (\gamma) & \cos \left(\gamma-\frac{2 \pi}{3}\right) & \cos \left(\gamma-\frac{4 \pi}{3}\right) \\
-\sin (\gamma)-\sin \left(\gamma-\frac{2 \pi}{3}\right) & -\sin \left(\gamma-\frac{4 \pi}{3}\right)
\end{array}\right]
$$

Compte tenu des relations (1) à (5), et en utilisant la représentation complexe $\left(\bar{G}_{\alpha \beta}=G_{\alpha}+j G_{\beta}\right)$, on obtient les équations de tensions dans le même repère $\alpha, \beta$ :

$$
\left\{\begin{array}{l}
\bar{v}_{\alpha \beta}=R \cdot \bar{i}_{\alpha \beta}+(L-M) \cdot \frac{d \bar{i}_{\alpha \beta}}{d t}+M \cdot \frac{d\left(\bar{i}_{\alpha \beta}+\bar{i}_{\alpha \beta}^{\prime}\right)}{d t}+\bar{e}_{\alpha \beta} \\
\bar{v}_{\alpha \beta}^{\prime}=R \cdot{\overline{i^{\prime}}}_{\alpha \beta}+(L-M) \cdot \frac{d \bar{i}_{\alpha \beta}^{\prime}}{d t}+M \cdot \frac{d\left(\bar{i}_{\alpha \beta}+\bar{i}_{\alpha \beta}^{\prime}\right)}{d t}+\bar{e}_{\alpha \beta}^{\prime}
\end{array}\right.
$$

où: $\quad R=r, L=l_{s}+\frac{m}{2}$ et $M=\frac{3}{2} \cdot m$

L'expression du couple électromagnétique s'écrit sous la forme :

$\Gamma=\frac{p}{\omega}\left(e_{\alpha} \cdot i_{\alpha}+e_{\beta} \cdot i_{\beta}+e_{\alpha}^{\prime} \cdot i_{\alpha}^{\prime}+e_{\beta}^{\prime} \cdot i_{\beta}^{\prime}\right)$

Pour les machines double étoile à fém. sinusoïdale, on note que $e_{\alpha}^{\prime}=e_{\alpha}$ et $e_{\beta}^{\prime}=e_{\beta}$. Ce constat permet d'une part de déduire du système d'équation (6) un schéma de type circuit pour les MSAP double-étoile sinusoÏdale à rotor lisse (Fig. 6) et d'autre part de simplifier l'expression du couple. Celle-ci devient alors :

$\Gamma=\frac{p}{\omega} \cdot\left[e_{\alpha} \cdot\left(i_{\alpha}+i_{\alpha}^{\prime}\right)+e_{\beta} \cdot\left(i_{\beta}+i_{\beta}^{\prime}\right)\right]$

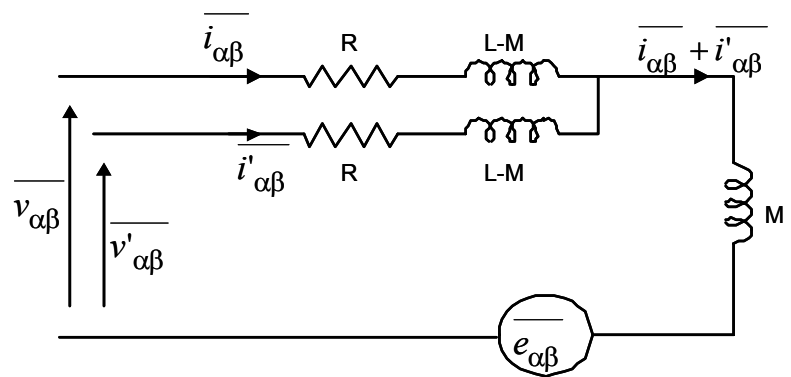

Figure 6 : Schéma de type circuit de MSAP double-étoile sinusoïdale à rotor lisse.

Nous remarquons que c'est uniquement la somme des courants, qui participe à la génération du couple. Pour cela, nous effectuons un changement de variables faisant apparaître la somme des courants et leurs différences. Nous utilisons alors la transformation suivante [1] :

$\left[\begin{array}{c}\bar{G}_{\alpha \beta}^{+} \\ \bar{G}_{\alpha \beta}^{-}\end{array}\right]=\frac{1}{\sqrt{2}}\left[\begin{array}{cc}1 & 1 \\ 1 & -1\end{array}\right] \cdot\left[\begin{array}{c}\bar{G}_{\alpha \beta} \\ \bar{G}^{\prime}\end{array}\right]$

En appliquant cette transformation au système d'équations (6), on aboutit à un système d'équations découplées entre elles :

$$
\begin{aligned}
& \bar{v}_{\alpha \beta}{ }^{+}=R \cdot \bar{i}_{\alpha \beta}{ }^{+}+(L+M) \cdot \frac{d \bar{i}_{\alpha \beta}{ }^{+}}{d t}+\bar{e}_{\alpha \beta}{ }^{+} \\
& \bar{v}_{\alpha \beta}{ }^{-}=R \cdot \bar{i}_{\alpha \beta}{ }^{-}+(L-M) \cdot \frac{d \bar{i}_{\alpha \beta}{ }^{-}}{d t}
\end{aligned}
$$

où :

$$
\begin{array}{llc}
\bar{e}_{\alpha \beta}{ }^{+}=\bar{e}_{\alpha \beta} \cdot \sqrt{2} \quad \text { et } & \bar{e}_{\alpha \beta}{ }^{-}=0 \\
L+M=l_{p}+2 . m & \text { et } & L-M=l_{p}-m
\end{array}
$$

L'expression du couple électromagnétique devient alors :

$\Gamma=\frac{p}{\omega} \cdot\left(e_{\alpha}^{+} \cdot i_{\alpha}^{+}+e_{\beta}^{+} \cdot i_{\beta}^{+}\right)$

Les relations (10) et (11) permettent d'obtenir les nouvelles composantes des courants statoriques :

- $\mathrm{i}_{\alpha}{ }^{+}$et $\mathrm{i}_{\beta}{ }^{+}$contiennent le fondamental du courant et certains harmoniques, et ils contribuent à la création du couple.

- $\mathrm{i}_{\alpha}{ }^{-}$et $\mathrm{i}_{\beta}{ }^{-}$contiennent d'autres harmoniques. Ces composantes créent des pertes Joule mais ne participent pas à la génération du couple.

Afin d'avoir une segmentation équilibrée de puissance, les tensions triphasées appliquées aux deux étoiles doivent avoir la même forme d'onde, mais être déphasées du même angle que le décalage entre les étoiles. Ce qui implique que les références de tensions des onduleurs à MLI qui les alimentent soient déphasées du même angle. Dans ces conditions, les composantes des tensions de la première étoile $\left(\mathrm{v}_{\alpha}, \mathrm{v}_{\beta}\right)$ ne sont pas instantanément identiques à celles de la seconde $\left(\mathrm{v}_{\alpha}^{\prime}, \mathrm{v}_{\beta}^{\prime}\right)$. Les composantes $\mathrm{v}_{\alpha}{ }^{-}$et $\mathrm{v}_{\beta}{ }^{-}$des tensions sont alors non-nulles et les courants correspondants $\left(i_{\alpha}{ }^{-}\right.$et $i_{\beta}{ }^{-}$) se manifestent sous forme des surintensités dans les courants de phases. Ces surintensités ont des amplitudes d'autant plus élevées que l'inductance de fuite est faible [1]. Dans le cas d'une MSAP à deux étoiles fortement couplées (L-M faible), décalées de $30^{\circ}$ électriques, la figure 7 présente les composantes $i_{\alpha}{ }^{-}$et $i_{\alpha}{ }^{+}$ des courants ainsi que le courant d'une phase et le couple généré. Compte tenu des fortes ondulations des composantes $i_{\alpha}{ }^{-}$(Fig. 7-a) et $i_{\beta}{ }^{-}$, le courant de phase (Fig. 7c) contient des pics non négligeables. On remarque qu'effectivement le couple n'est pas affecté par ces pics (Fig. 7-d), et ne contient que les ondulations dues aux composantes $\mathrm{i}_{\alpha}{ }^{+}$(Fig. 7-b) et $\mathrm{i}_{\beta}{ }^{+}$de courants (voir la relation 12). L'amplitude des ondulations de ces composantes de courants est faible car elle est limitée par l'inductance cyclique L+M (voir la relation 10).

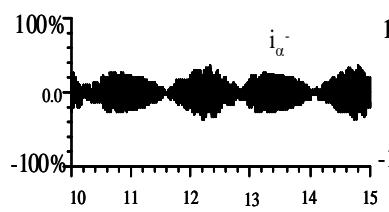

(a)

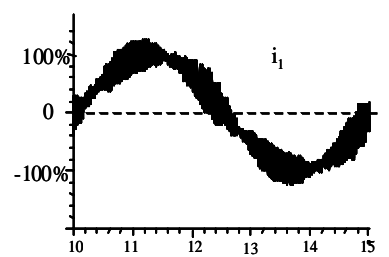

(c)

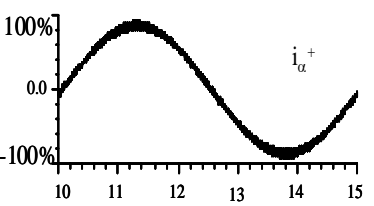

(b)

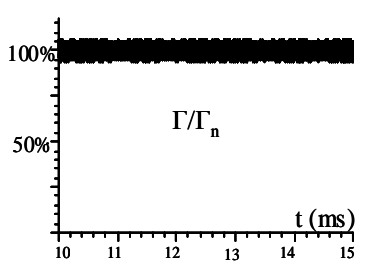

(d)

Figure 7 : Résultats concernant une MSAP à deux étoiles décalées $\left(\gamma=30^{\circ}\right)$ fortement couplées . 
Pour éviter l'apparition des surintensités, il est nécessaire que les tensions instantanées appliquées aux deux étoiles, ramenées dans le même repère ( $\alpha \beta$ par exemple), soient identiques. Cette condition ne peut être satisfaite que si le décalage $\gamma$ entre les étoiles est nul. Pour une machine à deux étoiles non décalées, alimentée par deux onduleurs à MLI, les figures 8-a et 8-b montrent respectivement la forme d'onde de courant et celle de couple. On constate qu'effectivement les courants ne comportent que les ondulations habituelles dues aux découpages, et le couple est presque identique à celui d'une machine à deux étoiles décalées.

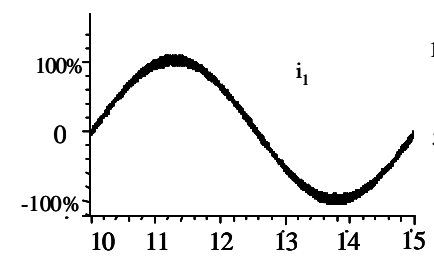

(a)

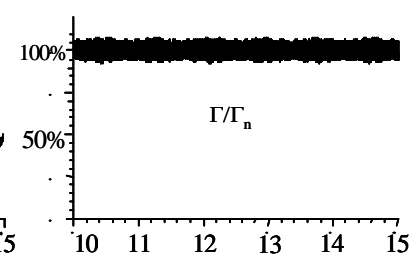

(b)
Figure 8 : Courant (à gauche) et couple (à droite) de la machine à deux étoiles non décalées $\left(\gamma=0^{\circ}\right)$.

Par conséquent, la sûreté de l'alimentation par deux onduleurs de tension d'une machine double-étoile est liée d'une part au décalage entre les étoiles, et d'autre part, au degré de couplage de ses étoiles.

Les machines à deux étoiles décalées et fortement couplées ne conviennent pas à une alimentation par onduleurs de tension. Dans ce cas, si l'on n'ajoute pas des inductances externes (volumineuses et lourdes) en série avec les enroulements de la machine, les courants de phases sont fortement ondulés. Il faut alors surdimensionner les composants des onduleurs et les condensateurs du filtrage de l'étage continu.

Pour les machines à deux étoiles non-décalées, alimentées par deux onduleurs de tension, on peut théoriquement éliminer les surintensités; compte tenu de l'imperfection de la commutation des composants, même en synchronisant la commande des onduleurs, il peut apparaître des retards de l'ordre de quelques microsecondes dans les tensions appliquées aux deux étoiles. De nouveau, les composantes $\mathrm{v}_{\alpha}{ }^{-}$et $\mathrm{v}_{\beta}{ }^{-}$des tensions réapparaissent. Cependant, compte tenu de faible retard entre les tensions appliquées aux deux étoiles, avec la même fréquence de découpage et la même tension de source continue, la valeur de l'inductance de fuite nécessaire à limiter les surintensités est beaucoup plus faible.

Par conséquent, la conception de machines double-étoile alimentée par deux onduleurs de tension doit prendre en compte le fait qu'une inductance de fuite minimale soit nécessaire au bon fonctionnement du système. Signalons seulement que dans le cas des machines synchrones à aimants permanents montés sur la surface du rotor, il est possible de réaliser deux étoiles non-décalées faiblement couplées [1]. En mode dégradé, avec une étoile nonalimentée, la forme de fém des phases de l'étoile restant active n'est pas modifiée, et la même qualité de couple qu'en mode normal peut être atteinte, sans changer la forme de courant dans les phases de l'étoile active.

\section{III.2 MSAP à trois phases séparément alimentées}

Les équations de tension et l'expression du couple de la machine synchrone à aimants (rotor lisse) à trois phases séparément alimentées (Fig. 4 ou Fig. 5) sont :

$$
\begin{aligned}
& \left(\begin{array}{l}
v_{1} \\
v_{2} \\
v_{3}
\end{array}\right)=R\left(\begin{array}{l}
i_{1} \\
i_{2} \\
i_{3}
\end{array}\right)+\left(\begin{array}{ccc}
L_{p} & M & M \\
M & L_{p} & M \\
M & M & L_{p}
\end{array}\right) \frac{d}{d t}\left(\begin{array}{c}
i_{1} \\
i_{2} \\
i_{3}
\end{array}\right)+\left(\begin{array}{c}
e_{1} \\
e_{2} \\
e_{3}
\end{array}\right) \\
& \Gamma=\frac{1}{\Omega} \sum_{j=1}^{3} e_{j} \cdot i_{j}
\end{aligned}
$$

où $v_{j}, i_{j}$ et $e_{j}$ sont la tension, le courant et la fém de la jème phase et $\Omega$ la vitesse angulaire du rotor; $R$ est la résistance des bobines statoriques, $L_{p}$ leur inductance propre et $M$ la mutuelle inductance négative entre deux bobines.

Le système d'équation (13) étant couplé, on le diagonalise en effectuant un changement de base adéquat. Les composantes d'un vecteur (courant, tension ou fém) dans la nouvelle base sont déduites des composantes de ce même vecteur dans l'ancienne base par l'application de la transformation de Concordia :

$\left(\begin{array}{l}g_{0} \\ g_{\alpha} \\ g_{\beta}\end{array}\right)=\left[T_{33}\right]^{t} \cdot\left(\begin{array}{l}g_{1} \\ g_{2} \\ g_{3}\end{array}\right)=\sqrt{\frac{2}{3}}\left[\begin{array}{ccc}\frac{1}{\sqrt{2}} & \frac{1}{\sqrt{2}} & \frac{1}{\sqrt{2}} \\ 1 & -\frac{1}{2} & -\frac{1}{2} \\ 0 & \frac{\sqrt{3}}{2} & \frac{\sqrt{3}}{2}\end{array}\right] \cdot\left(\begin{array}{l}g_{1} \\ g_{2} \\ g_{3}\end{array}\right)$

L'application de cette transformation conduit à la diagonalisation du système d'équation (13) :

$$
\begin{aligned}
& \left(\begin{array}{l}
v_{0} \\
v_{\alpha} \\
v_{\beta}
\end{array}\right)=R\left(\begin{array}{l}
i_{0} \\
i_{\alpha} \\
i_{\beta}
\end{array}\right)+\left[\begin{array}{ccc}
L_{0} & 0 & 0 \\
0 & L & 0 \\
0 & 0 & L
\end{array}\right] \frac{d}{d t}\left(\begin{array}{l}
i_{0} \\
i_{\alpha} \\
i_{\beta}
\end{array}\right)+\left(\begin{array}{l}
e_{0} \\
e_{\alpha} \\
e_{\beta}
\end{array}\right) \\
& \Gamma=\frac{1}{\Omega}\left[e_{0} \cdot i_{0}+\left(e_{\alpha} \cdot i_{\alpha}+e_{\beta} \cdot i_{\beta}\right)\right] \\
& \text { où : }\left[\begin{array}{ccc}
L_{0} & 0 & 0 \\
0 & L & 0 \\
0 & 0 & L
\end{array}\right]=\left(\begin{array}{ccc}
L_{p}+2 M & 0 & 0 \\
0 & L_{p}-M & 0 \\
0 & 0 & L_{p}-M
\end{array}\right)
\end{aligned}
$$

Dans une machine à répartition sinusoïdale des conducteurs des bobines statoriques, on a :

$$
\left\{\begin{array}{l}
L_{p}=l_{f}+m \\
M=m \cdot \cos \frac{2 \cdot \pi}{3}=-\frac{m}{2}
\end{array}\right.
$$

où $l_{f}$ est l'inductance de fuite et $\mathrm{m}$ est la mutuelle inductance entre deux bobines non décalées. En reportant (19) dans (18), on obtient :

$$
\left\{\begin{array}{l}
L_{0}=L_{p}+2 M=l_{f} \\
L=l_{f}+\frac{3}{2} \cdot m
\end{array}\right.
$$

Le système d'équations (16) contient 3 équations découplées. Ceci permet de définir deux machines électriques fictives équivalentes sans couplage magnétique entre elles $[6,8]$ : 
- L'une est monophasée et appelée machine homopolaire. Elle est modélisée par une résistance $\mathrm{R}$, une inductance $L_{0}$ correspondant à l'inductance de fuite de la machine et une fém $\mathrm{e}_{0}$ correspondant à la composante homopolaire du vecteur fém (Fig. 9-a). Celle-ci étant nulle dans les machines à fém sinusoïdale, le courant $i_{0}$ ne participe pas à la génération du couple $: \Gamma=\frac{1}{\Omega}\left(e_{\alpha} \cdot i_{\alpha}+e_{\beta} \cdot i_{\beta}\right)$

- L'autre est diphasée et appelée machine principale. Elle est modélisée par une résistance $\mathrm{R}$, une inductance $\mathrm{L}$ (inductance cyclique) et les fém $e_{\alpha}$ et $e_{\beta}$ (Fig. 9-b). Celles-ci sont sinusoïdales et déphasées de 90 degrés électriques dans les machines à fém sinusoïdale.

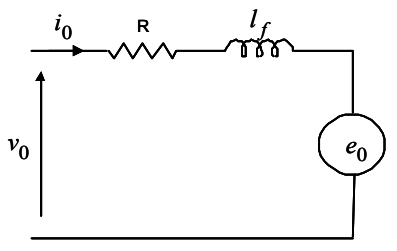

(a)

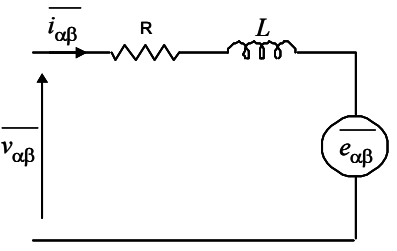

(b)
Figure 9 : Circuits équivalents de MSAP triphasée

Etant donné que dans les machines à fém sinusoïdale la composante homopolaire de la fém $\left(\mathrm{e}_{0}\right)$ est nulle, la composante homopolaire du courant ne participe pas à la génération du couple et crée uniquement des pertes; Afin de minimiser les pertes Joule, il faut donc forcer $i_{0}$ à zéro et alimenter uniquement la machine principale par des courants sinusoïdaux $i_{\alpha}$ et $i_{\beta}$ en phase avec les fém $e_{\alpha}$ et $e_{\beta}$. Autrement dit il faut imposer des courants de phases sinusoïdaux, en phase avec les fém correspondantes ; leur amplitude $\left(I_{M}\right)$ est proportionnelle au couple désiré :

$e_{n}(\theta)=-K_{e} \Omega \cdot \sin (\theta-(n-1) 2 \pi / 3)$

$i_{n}(\theta)=-I_{M} \cdot \sin (\theta-(n-1) 2 \pi / 3)$

$\Gamma=\frac{3}{2} K_{e} \cdot I_{M}$

où $\mathrm{K}_{\mathrm{e}}$ est une constante des MSAP sinusoïdales.

Les trois phases de la machine étant alimentées séparément, trois boucles de contrôle sont nécessaires pour imposer les courants. Indépendamment du régulateur choisi, chaque onduleur monophasé peut imposer trois niveaux de tension ( $v_{j}=\mathrm{U}_{0}, 0$ ou $-\mathrm{U}_{0} ; \mathrm{U}_{0}$ étant la tension de la source continue). Les trois onduleurs ne peuvent donc imposer que $3^{3}$ vecteurs tension $\left(\left[\begin{array}{lll}v_{1} & v_{2} & v_{3}\end{array}\right]^{t}\right)$ distincts dont seuls sept sont à composante homopolaire nulle [6]. Par conséquent, la commande indépendante des onduleurs monophasés conduit à imposer aléatoirement à la machine des tensions $\left[v_{1} v_{2} v_{3}\right]^{t}$ à composantes homopolaires positives, nulles ou négatives. La machine fictive homopolaire est alors excitée et le courant correspondant $\left(\mathrm{i}_{0}\right)$ se manifeste sous forme des surintensités dans les courants de phases. Ces surintensités ont des amplitudes d'autant plus élevées que l'inductance de fuite est faible ( $l_{f}$ dans Fig. 9-a). Dans le cas d'une MSAP triphasée à faible inductance de fuite, la figure 10 présente les composantes $i_{0}, i_{\alpha}$ et $i_{\beta}$ des courants ainsi que le courant d'une phase et le couple. Compte tenu des fortes ondulations de $\mathrm{i}_{0}$ (Fig. 10-c), le courant de phase (Fig. 10-a) a un taux d'ondulation élevé. Ce taux élevé augmente considérablement les pertes et nécessite un surdimensionnement des différents composants de la chaîne de conversion électromécanique d'énergie. On remarque que le couple (Fig. 10-b) n'est pas affecté par les fortes ondulations des courants de phases, et ne contient que les ondulations dues aux composantes $i_{\alpha}$ et $i_{\beta}$ (Fig. 10-d) du courant (relation 21).

Pour réduire l'amplitude des ondulations du courant, une solution consiste à exciter la machine fictive homopolaire par des tensions aussi faibles que possible ; cela a conduit à la définition d'une stratégie de commande privilégiant l'application des vecteurs tension à composante homopolaire nulle [6]. Cependant, compte tenu de l'imperfection de la commutation des composants, pour limiter le taux d'ondulation de courant, il est nécessaire de concevoir des MSAP à inductance de fuite suffisante pour l'application considérée.

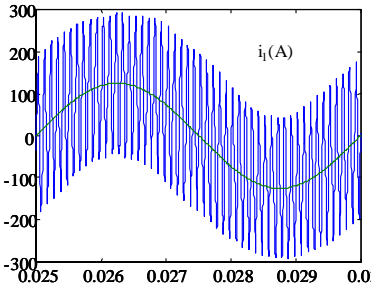

(a)

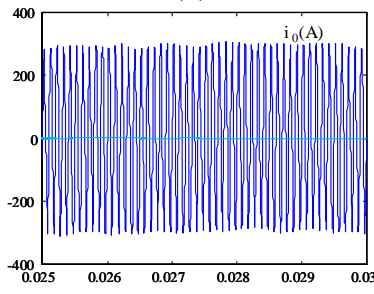

(c)

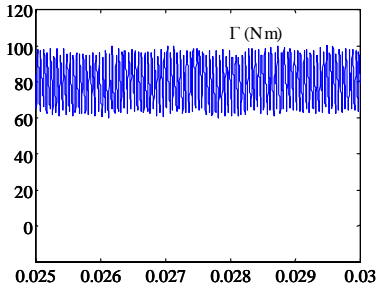

(b)

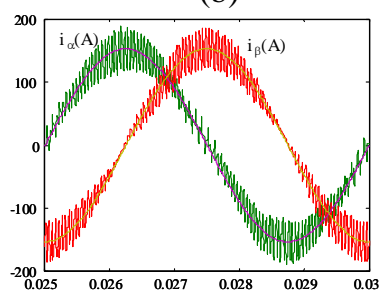

(d)
Figure 10 : Les formes du courant de phase (a), du couple (b), des composantes homopolaire (c) et $\alpha-\beta$ (d) du courant (MSAP triphasée à faible inductance de fuite).

En cas de défaut d'un onduleur monophasé, conduisant à l'annulation du courant dans la jème phase, la machine continue à développer un couple moyen non-nul :

$$
\Gamma_{d}=\frac{K_{e} \cdot I_{M}}{2}\left(2+\cos \left(2 . \theta-2(j-1) \frac{2 . \pi}{3}\right)\right)
$$

Sans modification des courants dans les phases actives, il apparaît des ondulations de couple d'amplitude élevée et de fréquence basse. Une solution simple et efficace, basée sur les méthodes de filtrage du couple des MSAP polyphasées [2], consiste à modifier le courant dans l'une des deux phases actives (par exemple $\mathrm{m}^{\text {ème }}$ ) comme suit :

$i_{m f}(t)=I_{M} \cdot \sin \left(\theta-(j-1) \frac{2 \cdot \pi}{3}\right)$

L'expression du couple filtré en mode dégradé devient :

$\Gamma_{d f}=\frac{K_{e} \cdot I_{M}}{2}\left(1+2 \cdot \cos ^{2}\left((m-j) \frac{2 . \pi}{3}\right)\right)=\frac{3}{4} K_{e} \cdot I_{M}$

En mode dégradé, sans modifier l'amplitude des courants dans les phases actives, la MSAP à trois phases développe la moitié du couple en mode normale. 
La machine à aimants triphasée, alimentée par trois onduleurs monophasés de tension, et la MSAP à deux étoiles alimentées par deux onduleurs triphasés de tension ont plusieurs points communs :

- Dans les deux cas, l'utilisation des machines à faible couplage magnétique, entre les phases ou entre les étoiles, facilite la commande, réduit les pertes et améliore la sûreté de fonctionnement.

- Les onduleurs alimentant les deux machines ont le même nombre de composants et sont soumis aux mêmes contraintes en courant et en tension.

- Les deux structures, peuvent fonctionner en mode dégradé, en l'absence d'alimentation d'une phase ou d'une étoile. A courant maximal imposé, le couple filtré dans les deux cas est la moitié du couple en mode normal.

Cependant, les points suivants distinguent les deux structures :

- La tension nominale de la machine triphasée est double de celle de la machine double-étoile mais la valeur nominale de leurs courants est la même; la machine triphasée peut être réalisée en mettant en série les bobines homologues des deux étoiles non-décalées d'une machine double-étoile (Fig. 11).

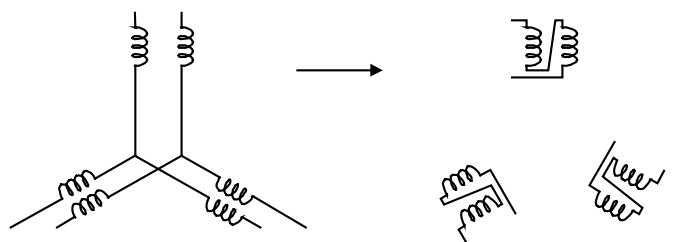

Figure 11 : Bobinage double-étoile et le bobinage triphasé correspondant.

- En mode normal, pour le contrôle du couple d'une machine double-étoile quatre boucles de courant (quatre capteurs de courant) sont nécessaires; tandis qu'il en faut trois avec une machine triphasée (un capteur de moins).

- En mode dégradé, il faut modifier la forme du courant de l'une des deux phases actives de la machine triphasée tandis qu'avec une machine double-étoile la forme de courant des phases de l'étoile restant active n'est pas modifiée. IV- Extension de la segmentation de puissance à la
source électrique

Les onduleurs de tension alimentant des MSAP étudiées au paragraphe précédent sont connectés au même bus continu (figures 3 à 5). Ce bus est alimenté via un ensemble redresseur-filtre LC à partir d'un réseau triphasé. Lorsqu'il existe deux réseaux isolés électriquement, on peut envisager d'étendre la segmentation de puissance, limitée jusqu'ici à l'ensemble onduleur-machine, à la source électrique. Si les réseaux d'avion sont triphasés, on doit utiliser deux redresseurs et deux filtres LC pour créer les deux bus continus isolés. Cet inconvénient disparaît naturellement dans le cas où l'énergie électrique serait distribuée par deux réseaux isolés continus; le remplacement des réseaux triphasés d'avion par des réseaux continus diminue également d'autres contraintes liées à la variation de tension et de fréquence des réseaux triphasés.

Dans le cas des deux structures étudiées au paragraphe précédent, nous analysons ci-après les conséquences de la segmentation de puissance au niveau de la source continue.

\section{IV.1 MSAP double-étoile alimentée par deux sources continues via deux onduleurs de tension}

La figure 12 présente l'architecture segmentée d'alimentation d'une MSAP double-étoile par deux réseaux triphasés électriquement isolés. On remarque que dans le cas général, les deux sources continues, créées par le redressement des tensions de deux réseaux indépendants, ont des tensions de valeurs plus ou moins différentes ( $U_{01} \neq U_{02}$ sur la Fig.12). Les tensions appliquées par les onduleurs aux deux étoiles n'ont donc pas instantanément les mêmes valeurs; l'écart entre ces tensions est d'autant plus élevé que la différence $\left|U_{01}-U_{02}\right|$ est plus importante. Compte tenu du modèle de la MSAP double-étoile et son circuit équivalent (\$III.1, Fig. 6), pour éviter un taux d'ondulation élevé des courants de phase, l'inductance de fuite entre les étoiles doit avoir une valeur suffisante. A fréquence de découpage imposée, cette valeur d'inductance de fuite devrait être supérieure à celle nécessaire pour limiter le courant de circulation entre deux onduleurs connectés à la même source continue (Fig. 3). Pour éviter cette contrainte supplémentaire, il est possible de connecter les deux redresseurs au même réseau triphasé, dès que la différence des tensions des deux sources continues dépasse une valeur limite $\Delta U_{0_{\max }}$ fixée a priori $\left(\left|U_{01}-U_{02}\right|>\Delta U_{0 \text { max }}\right)$. De préférence, le réseau dont l'amplitude de la tension est plus élevée est sélectionné. Cette stratégie d'alimentation résout automatiquement le problème d'apparition de défauts sur l'un ou l'autre réseau.

L'extension de la segmentation de puissance à la source continue, au prix de l'ajout d'un deuxième ensemble redresseur-filtre, permet d'assurer le fonctionnement de l'actionneur en cas de défaut interne de l'un des deux ensembles redresseur-filtre.

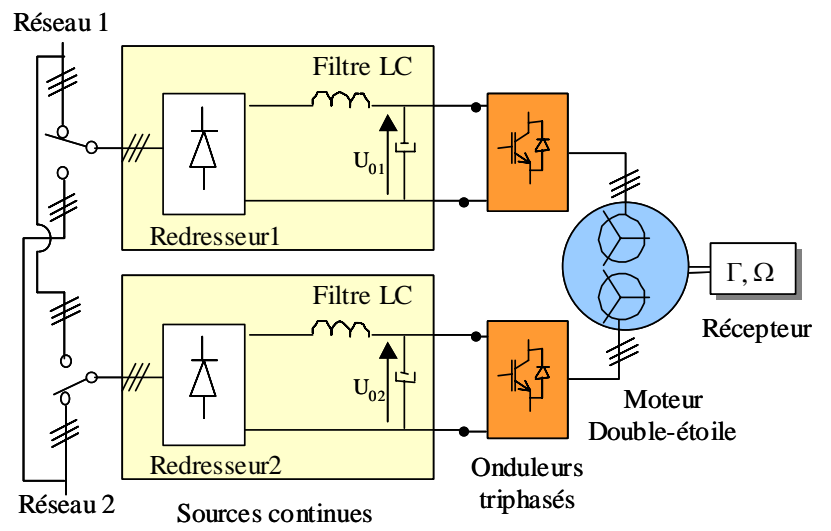

Figure 12 : MSAP double-étoile alimentée par deux onduleurs de tension connectés à deux sources continues. 
Remarque : En surdimensionnant les onduleurs et les redresseurs, le fonctionnement à puissance nominale avec une étoile alimentée est envisageable sur une durée courte. En effet, certains actionneurs d'avion ne sont activés que durant un intervalle court, n'entraînant pas un échauffement excessif de la machine. Dans ce cas, si le couplage magnétique entre les étoiles de la machine est suffisamment faible, un tel actionneur peut être considéré comme équivalent à deux actionneurs mono-convertisseur mono-machine entraînant la même charge mécanique. Ainsi, en présence de défauts sur une chaîne de conversion d'énergie (constituée d'un redresseur, d'un filtre LC, d'un onduleur et d'une étoile), l'autre chaîne de conversion permet un fonctionnement presque normal. Notons aussi que le volume d'une machine double étoile est moins important que celui de deux machines de puissance moitié ; cette différence de volume justifie l'utilisation d'une machine avec une alimentation segmentée à la place de deux machines triphasées de moitié de puissance.

\section{IV.2 MSAP triphasée alimentée par deux sources continues via onduleurs de tension}

La figure 13 présente l'architecture segmentée d'alimentation d'une MSAP triphasée par deux réseaux électriquement isolés : le redresseur 1 (resp. 2) est alimenté par le réseau 1 (resp. 2). Comme nous l'avons mentionné au $\S$ IV.1, les deux sources continues, créées par le redressement des tensions de deux réseaux indépendants, ont des tensions de valeurs plus ou moins différentes. Avec cette architecture d'alimentation par des réseaux isolés, les sources continue isolées $U_{01}$ et $U_{02}$ sont mises en série par l'intermédiaire des interrupteurs des deux onduleurs et les bobines de la machine. Par conséquent, il n'y a pas de contrainte supplémentaire liée à la différence des tensions des sources continues. De plus, avec les sources isolées, la somme des courants de phases et donc la composante homopolaire du courant et les pertes correspondantes sont nulles. En l'absence de défaut d'onduleur, on peut aussi réduire considérablement les pertes à basse vitesse; en effet, cette architecture d'alimentation permet d'appliquer un vecteur tension nul par un onduleur triphasé et de contrôler les courants et donc le couple à l'aide de l'autre onduleur. Au-delà d'une certaine vitesse, les deux onduleurs sont pilotés par des commandes complémentaires afin de doubler la tension appliquée à chaque phase de la machine.

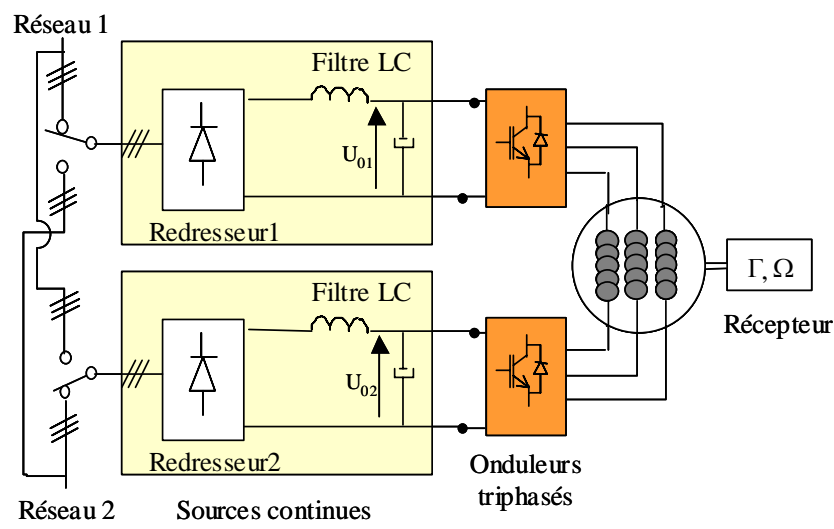

Figure 13 : MSAP triphasée alimentée par deux onduleurs de tension connectés à deux sources continues.
En présence de défauts, la stratégie la mieux adaptée est fortement dépendante de la nature du défaut et de sa localisation, et dépasse le cadre de cet article. Néanmoins, on peut également envisager des disjoncteurs supplémentaires pour reconfigurer soit l'architecture d'alimentation soit la connexion des trois bobines de la machine.

\section{Conclusion}

Les machines synchrones à aimants permanents, doubleétoile ou triphasée, à faible couplage magnétique entre les étoiles ou entre les phases, autorisent une alimentation segmentée par onduleurs de tension sans qu'il y ait la nécessité d'éléments inductifs externes. Avec ces structures, un onduleur de tension triphasé est remplacé par deux onduleurs de tension modulaires utilisant des interrupteurs de calibre réduit (courant ou tension). Cela permet d'apporter une souplesse considérable par la possibilité d'utiliser des composants soit fabriqués en grand nombre (coût), soit plus fiables ("mono-chip"), soit à caractéristiques spécifiques (haute température). De plus, en cas de défaut d'un onduleur, l'actionneur peut continuer à fonctionner en mode dégradé grâce à l'autre onduleur actif.

Pour les deux structures étudiées, l'extension de la segmentation de puissance à la source continue, au prix de l'ajout d'un deuxième ensemble redresseur-filtre, accroît le degré de fiabilité de l'actionneur. En mode normal de fonctionnement, l'alimentation des machines triphasées par deux sources isolées, réduit sensiblement leurs pertes. En présence de défauts sur l'une des deux chaînes d'alimentation d'une machine double-étoile, l'autre chaîne permet un fonctionnement presque normal.

\section{Références}

[1] N. Moubayed, F. Meibody-Tabar, B. Davat, "Alimentation par deux onduleurs de tension d'une machine synchrone double étoile", Revue Internationale de Génie Electrique, vol. 1, n 4, 1998, pp. 457-470.

[2] J-P. Martin, F. Meibody-Tabar, B.Davat, "Multiple-phase permanent magnet synchronous machine supplied by VSIs working under fault conditions", IEEE-IAS annual meeting, Roma, october 2000, CDROM

[3] A. Bouscayrol, B. Davat, B. de Fornel, B. François, J. P. Hautier, F. Meibody-Tabar, M. Pietrzak-David, "Multi-machine multi-converter systems: applications to electromechanical drives", EPJ Applied Physics, Vol. 10, no. 2, May 2000, pp. 131-147.

[4] A. Bouscayrol, B. Davat, P. Escané, B. de Fornel, B. François, D. Hadiouche, J-P. Hautier, J-P. Martin, F. Meibody-Tabar, E. Monmasson, V. de Olivera, R. Pena-Eguiluz, J. Pierquin, M. Pietrzak-David, H. Razik et E. Semail, "Structures de commande des systèmes multi-machines multi-convertisseurs", RIGE, vol. 6, n 3-4, 2003, pp 307-324.

[5] T. Gopalarathnam, H. A. Toliyat, J. C. Moreira, "Multi-phase faulttolerant brushless DC motor drives", Conf. Rec. IEEE - IAS Annual Meeting, Roma (Italy), Oct. 2000.

[6] J-P. Martin, E. Semail, S. Pierfederici, A. Bouscayrol, F. MeibodyTabar et B. Davat, "Space vector control of 5-phase PMSM supplied by 5 H-bridge VSIs", Conference on Modeling and Simulation of Electric Machines, Converters and Systems (ElectrIMACS'02), 1821 août 2002, Montreal (Canada), Cd-Rom.

[7] T. M. Jahns, "Improved reliability in solid-state AC drives by means of multiple independent phase-drive units", IEEE Transactions on Industry Applications, 1980, vol. IA-16, n 3, pp. 321-331.

[8] E. Semail, "Outils et méthodologie d'étude des systèmes électriques polyphasés. Généralisation de la méthode des vecteurs d'espace", Thèse de Dctorat de l'USTL, 2000, Lille (France). 